\title{
Disfunções posturais no uso dos laptops relacionado à sintomatologia dolorosa sobre a coluna vertebral
}

\author{
Juscelino Francisco Vilela Junio ${ }^{1}$, Anna Luísa Araújo Brito², Juceluce da Silva Vilela ${ }^{3}$, \\ Luiz Henrique Ferreira de Aguiar ${ }^{4}$, Emerson Nunes da Silva ${ }^{5}$, Eurico Solian Torres Liberalino ${ }^{6}$
}

\begin{abstract}
RESUMO
O presente estudo objetivou analisar a utilização de uso dos laptops e sua relação com sintomatologia dolorosa sobre a coluna vertebral. Foi realizada uma entrevista com 246 acadêmicos de uma instituição de ensino superior do estado de Pernambuco. A maior parte dos entrevistados relatou desconforto musculoesquelético na coluna lombar $(42,6 \%)$ e coluna cervical $(36,1 \%)$, onde o maior tempo de utilização dos computadores portáteis nos acadêmicos está entre 1 a 2 horas (39,6\%). Dentre o posicionamento adotado para utilização dos notebooks, o mais comum é o sentado com o notebook sobre a mesa $(40,2 \%)$ e em segundo lugar, a posição de decúbito dorsal (19,9\%). E quanto à realização de alongamentos e/ou pausas durante a utilização do notebook, os resultados mostram que $8,1 \%$ são adeptos destas práticas, $65,8 \%$ não realizam e $26,4 \%$, esporadicamente. Os resultados sugerem uma provável associação entre dores na coluna e a postura de utilização dos computadores portáteis, logo se torna importante criar uma ergonomia para esse tipo de aparelho, assim como também é crucial uma corresponsabilidade em adotar hábitos posturais biomecanicamente corretos.
\end{abstract}

Descritores: Ergonomia; Postura; Dor

\section{Dysfunctions postural use of laptops related to painful symptomatology on the spine}

\begin{abstract}
This study aimed to analyze whether use of laptops and its relationship to pain symptoms on the spine. was conducted an interview with 246 students of a higher education institution in the state of Pernambuco. Most respondents reported musculoskeletal discomfort in the lumbar spine (42.6\%) and cervical spine (36.1\%), where the longer use of laptop computers in academic is between $1-2$ hours $(39.6 \%)$. Among the positioning adopted for use of notebooks, the most common is sitting with notebook on the table $(40.2 \%)$ and secondly, the supine position $(19.9 \%)$. And on the achievement of stretching and/or breaks while using the notebook, the results show that $8.1 \%$ are supporters of these practices, $65.8 \%$ and $26.4 \%$ do not perform sporadically. The results suggest a possible association between back pain and posture for the use of laptop computers, soon becomes important to create an ergonomic for this type of device, as well as a shared responsibility to adopt biomechanically correct postural habits is also crucial.
\end{abstract}

Descriptors: Ergonomics; Posture; Pain.

${ }^{1}$ Graduado em Fisioterapia pela Associação Caruaruense de Ensino Superior (ASCES), Caruaru, PE, Brasil.

${ }^{2}$ Graduada em Fisioterapia pela Associação Caruaruense de Ensino Superior (ASCES), Caruaru, PE, Brasil.

${ }^{3}$ Especialista em Ensino da Biologia e Novas Tecnologias pela Faculdade de Formação de Professores de Belo Jardim (FABEJA), Belo Jardim, PE, Brasil.

${ }^{4}$ Graduado em Educação Física pela Associação Caruaruense de Ensino Superior (ASCES), Caruaru, PE, Brasil.

${ }^{5}$ Graduando em Educação Física na Associação Caruaruense de Ensino Superior (ASCES), Caruaru, PE, Brasil.

${ }^{6}$ Especialista em Fisioterapia na Terapia Intensiva pela Faculdade Maurício de Nassau (FMN/CG), Campina Grande, PB, Brasil. 


\section{Introdução}

Atualmente os aparelhos de informática tornaram-se indispensáveis no cotidiano independente de ser no trabalho ou entretenimento, contribuindo para agilizar atividades do dia a dia. Por estes motivos, surgiram os computadores portáteis, atendendo às necessidades dos usuários que necessitavam do trabalho informatizado fora do seu âmbito de localidade, assim facilitando 0 acesso à internet e troca de informações em tempo real ${ }^{1,2}$.

No entanto esses aparelhos foram projetados para uso de curta duração, não favorecendo o conforto durante longas jornadas de trabalho. Os mesmos apresentam vantagens como processadores de baixo consumo, portabilidade, menor espaço de ocupação e processamento de informações mais rápido do que os computadores convencionais, seu uso substituiu os antigos computadores de mesa. Porém, apresenta desvantagens para 0 uso de longa duração como a limitação de adaptação ergonômica decorrente ao monitor, o qual é unido ao teclado, fazendo com que o usuário permanece com a cabeça anteriorizada para melhor visualização da tela durante 0 ato de digitação na postura sentado. Outro fator desencadeador de alterações na postura é a portabilidade que permite ao usuário colocar o aparelho em várias posturas ${ }^{1-3}$.

Quando os notebooks são utilizados de maneira exagerada e em posturas inadequadas, podem predispor os usuários a apresentarem sintomatologia de dores musculoesquelética e alterações na biomecânica da coluna vertebral. Com o crescimento da utilização desses aparelhos, torna-se necessário a realização de estudos ergonômicos voltados para a utilização correta desses dispositivos, com o objetivo de prevenir danos sobre as estruturas do corpo, uma vez que a sobrecarga gerada sobre o sistema musculoesquelético pode desencadear desde sintomatologia dolorosa até incapacidades funcionais. Como contramedida a esses danos, existe a intervenção da ergonomia que visa livrar os usuários de problemas relacionados à saúde ${ }^{4-10}$.

A ergonomia busca a adaptação de um local de trabalho ou objeto aos seus usuários, aborda ainda uma visão sobre as possiveis complicações que o uso indevido pode causar. Dessa forma, com a crescente expansão e necessidade de uso, é relevante abordar os aspectos ergonômicos dos Laptops, pois, a portabilidade desses aparelhos oportuniza a utilização em várias posições, implicando assim na adoção de posturas confortáveis, porém biomecanicamente erradas, que cronicamente se mantidas podem levar a dores e alterações posturais, repercutindo sobre a qualidade de vida dos usuários ${ }^{11-13}$.

O fato de ser um aparelho que pode gerar danos sobre a saúde quando mal utilizado, está relacionado aos aspectos físicos desse tipo de aparelho, que limita a adaptação ao usuário, por ter a tela acoplada ao teclado ${ }^{11}$. Essa falta de ajustes ergonômicos que os notebooks apresentam, proporciona uma disputa relacionada a portabilidade, conveniência e posturas confortáveis, muitas vezes levando o indivíduo a permanecer em posicionamentos estáticos por longos períodos, assim adotando posturas inadequadas. Posições fixas mantidas por tempo prolongado ou agressões externas ao aparelho osteomuscular relacionado às atividades monótonas e repetitivas geram uma diminuição local do fluxo sanguíneo e da oxigenação tecidual, causando fadiga e dor. Essas sintomatologias são decorrentes das tentativas de os usuários buscarem posturas confortáveis durante a utilização, que dependente do tipo, amplitude, intensidade e frequência dos movimentos, podem ser a gênese de vários distúrbios $23,3,11,14$.

Entre os riscos relacionados ao uso incorreto dos laptops, pode-se destacar à alteração sobre a biomecânica da coluna cervical, condição gerada pela maior flexão da coluna cervical realizada na utilização dos notebooks, no qual o usuário se posiciona devido ao ângulo da tela em relação à visão do mesmo. Quando o indivíduo é exposto cronicamente a esse posicionamento pode referir sintomas na região cervical como dor, limitação da amplitude dos movimentos fisiológicos, sensibilidade ou dor a palpação dos músculos cervicais, alterações posturais e até disfunções temporomandibulares ${ }^{15-17}$.

Com a crescente utilização dessa tecnologia em todas as faixas etárias, é de grande relevância conhecer as implicações que o uso indevido desses aparelhos pode trazer a saúde dos usuários e junto a isso propor medidas que minimizem esses efeitos, pois, a utilização desses aparelhos atinge um grande contingente da população geral, assim, da mesma maneira que cresce o acesso a informatização cresce também o uso inadequado dos aparelhos. 


\section{Materiais e métodos}

Trata-se de um estudo descritivo, exploratório e transversal, com abordagem quantitativa. Realizado em uma instituição de ensino superior do estado de Pernambuco que oferece cursos de graduação e pós-graduação em diversas áreas.

Para realização da revisão da literatura, foi utilizado as seguintes palavras chaves, de forma combinadas em pares e sistemática, pelo buscador booleano "AND": ergonomia, coluna vertebral, educação postural, laptop (ergonomics, spine, postural education, laptop).

Para o cálculo do tamanho amostral adotou-se os seguintes parâmetros: intervalo de confiança de 95\%; erro máximo tolerável de 2 pontos percentuais; efeito do desenho (deff) $=2$; e, por se tratar de um estudo abrangente com análise de múltiplos fatores sobre postura e aspectos ergonômicos, sendo estes voltados a utilização continua de laptops e postura inadequada, definiu-se a prevalência estimada em 50\%. Adicionalmente, visando atenuar as limitações impostas por eventuais perdas na aplicação e/ou preenchimento inadequado dos questionários, decidiu-se por acrescer em $20 \%$ o tamanho da amostra, totalizando em 246 pesquisados.

A coleta de dados foi realizada através de entrevista estruturada e método de amostra por conveniência, no período de 21 de outubro de 2013 a 28 de fevereiro de 2014, no Campus II da instituição pesquisada nos três turnos. Foi utilizado como critério de inclusão os universitários matriculados na instituição que utilizavam notebooks com tempo mínimo de 60 minutos por dia.

$\mathrm{O}$ instrumento da pesquisa foi um questionário adaptado do estudo de Pinheiro ${ }^{18}$, contendo as seguintes variáveis: sexo, idade, atividade física, tempo de utilização do equipamento (notebook), posicionamento do notebook e localização de maior desconforto. Consiste em uma série ordenada de 6 perguntas fechadas, necessitando ser preenchido em cada questão com um "x" na caixa apropriada.

Os dados foram tabulados e analisados pelo programa Epi Data (versão 3.1) nos formatos arquivo de texto (extensão TXT) e, posteriormente, documento (extensão QES). O recurso CHECK foi utilizado para controlar eletronicamente a entrada dos dados coletados na fase de digitação. Com o intuito de dar mais fidedignidade às respostas dos entrevistados e prevenir erros, os dados foram redigitados em outro computador, por outro pesquisador, e submetidos ao programa "VALIDATE" do Epi Data, sendo gerado um arquivo contendo informações sobre os erros de digitação, a fim de corrigi-los e orientar o processo de revisão e limpeza do banco de dados.

A pesquisa foi desenvolvida após a aprovação pelo comitê de ética, através do número (454.323), atendendo aos preceitos da resolução $n^{\circ}$ 466/12, do Comitê Nacional de Ética e Pesquisa em Seres Humanos. Foi mantido sigilo sobre os dados dos participantes da pesquisa, os quais assinaram um termo de Consentimento Livre e Esclarecido (TCLE), o qual foi disponibilizado em duas vias, ficando uma com o pesquisador e outra com o sujeito pesquisado. A mesma não envolveu nenhum tipo de intervenção ou procedimento invasivo, representando, portanto, risco mínimo, atendo-se somente, a perguntas do questionário respondido do entrevistado em sua rotina de aula e um possível constrangimento frente aos questionamentos, para o qual se buscou solução, executando em local reservado para proporcionar ao pesquisado mais comodidade. Como maneira de beneficiar os pesquisados, foram dadas orientações e esclarecido dúvidas sobre a maneira correta de utilização do computador portátil.

\section{Resultados}

Quando os pesquisados foram questionados sobre a realização de atividades físicas, o estudo mostrou que apenas $41,1 \%$ dos participantes realizam-nas. No que se refere à realização de alongamentos e/ou pausas durante a utilização do notebook, os resultados mostram que $8,1 \%$ são adeptos destas práticas, $65,8 \%$ não realizam e $26,4 \%$, esporadicamente.

As figuras a seguir mostram respectivamente, dados referentes à região de desconforto, tempo de utilização e posicionamento mais utilizados. 
Figura 1 - Região de desconforto musculoesquelético

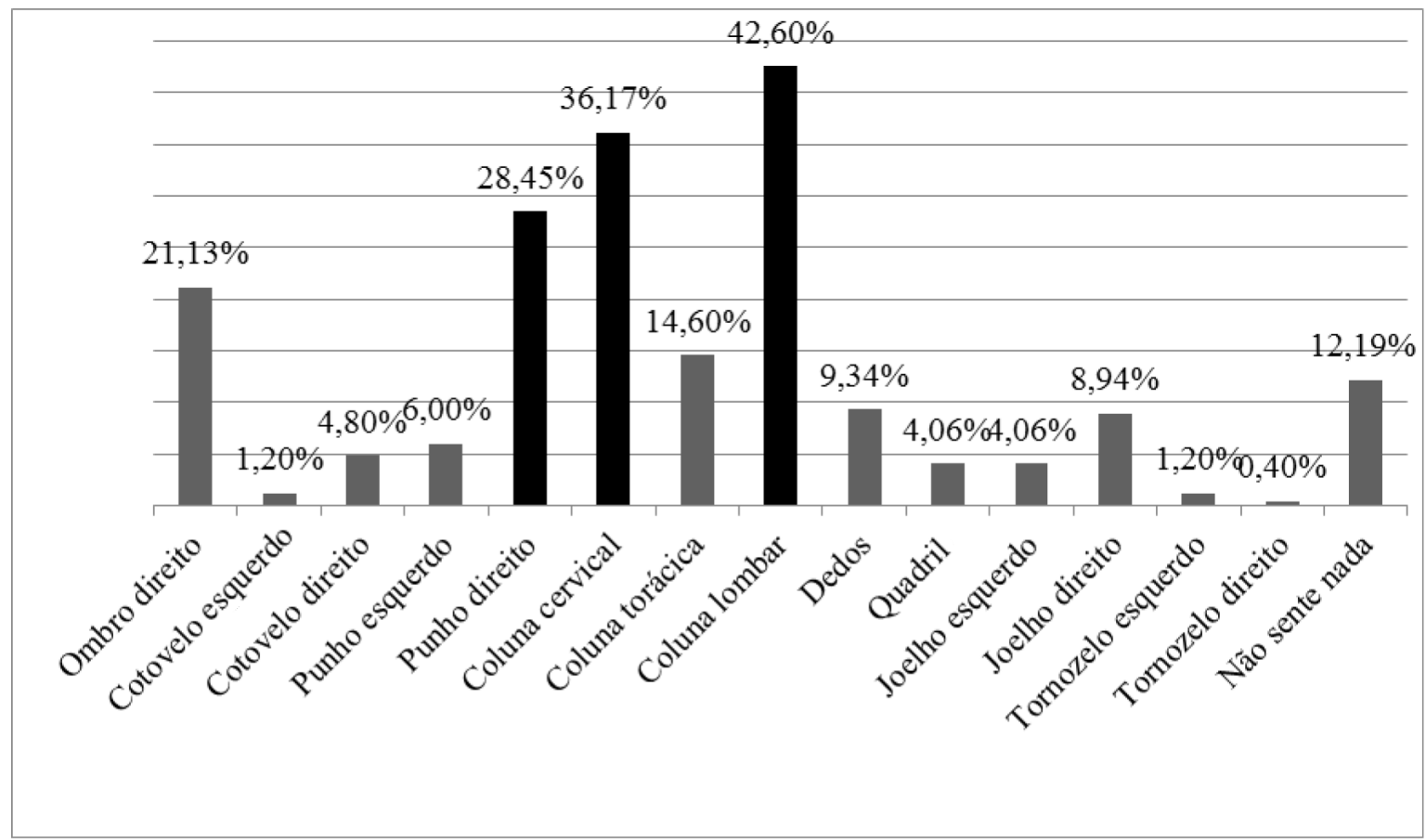

O presente estudo revelou a existência de múltiplas dores, entre as de maior destaque, revelam-se da coluna lombar e cervical, como apresentado na figura 1, e essas dores, podem ter relação com o uso prolongado dos computadores portáteis. Uma vez que esses aparelhos tornaram-se indispensáveis ao cotidiano, sua utilização cresceu de tal forma, que faz parte do convívio de todas as faixas etárias. Desta forma, quando esses dispositivos informatizados são utilizados de maneira exorbitante, podem afetar adversamente músculos do pescoço, parte superior das costas, ombros e braços, levando a fadiga muscular $3,4,10,15,18-20$.

Quando posturas erradas são mantidas por longos períodos de maneira estática ou são submetidas a excesso do ciclo de repetição de cada atividade, podem gerar maiores níveis de falha muscular, levando a quadros de fadiga, repercutindo em diminuição da força, velocidade de contração e da potência muscular. Ocasionando diminuição da performance, que se apresenta primeiro por desequilíbrios metabólicos, e algumas horas ou dias depois pela instalação de um processo inflamatório, relacionado à lesão muscular. Recentes estudos abordam que a inatividade física pode ser um agravante para a ocorrência de desconforto e lesão musculoesquelética, relacionada à diminuição da flexibilidade e oxigenação tecidual. Concordando com a literatura os resultados desse estudo aponta um significativo percentual de pessoas inativas, medida que pode ser um importante ampliador das queixas de dores ${ }^{21-23}$.

Figura 2 - Tempo de utilização do notebook

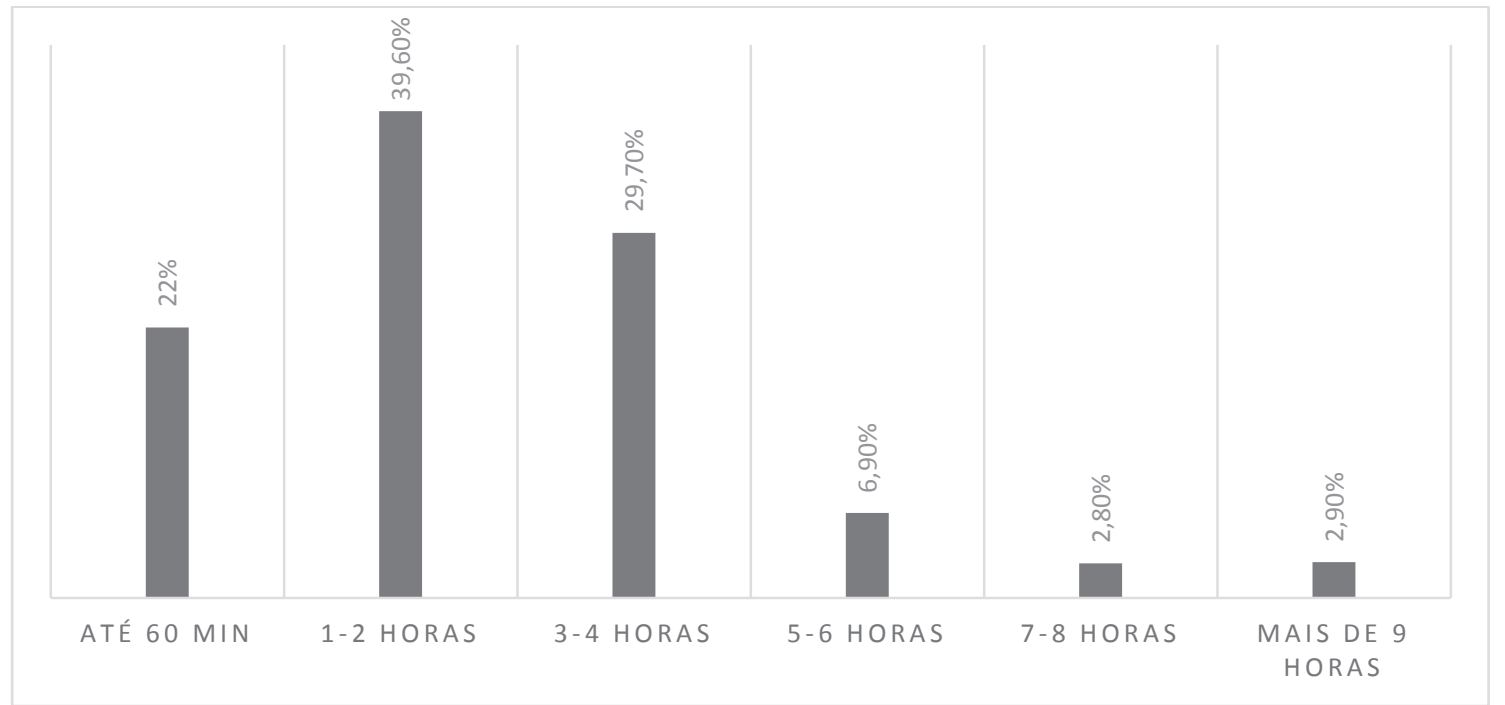


No que se refere aos agentes que podem aumentar os riscos de lesões musculoesqueléticos e danos sobre a coluna vertebral, é importante abordar a falta de pausas e alongamentos, como um fator de grande influência no surgimento de desconfortos. Quando são associados ao uso indevido do notebook, somado ao esforço excessivo de atividades, posições impróprias do corpo e longos períodos de utilização, acumulam lentamente prejuízos para o corpo e podem provocar lesões em várias regiões, tais como, mãos, punhos, braços, ombros e pescoço, as quais acabam se transformando numa condição grave e muito dolorosa que não se apresentam de imediato. São doenças que vem ao longo do tempo e que aumentam o risco se o usuário utilizar o computador por seguidas horas e diariamente ${ }^{18,22}$.

Figura 3 - Posicionamento de utilização dos computadores portáteis

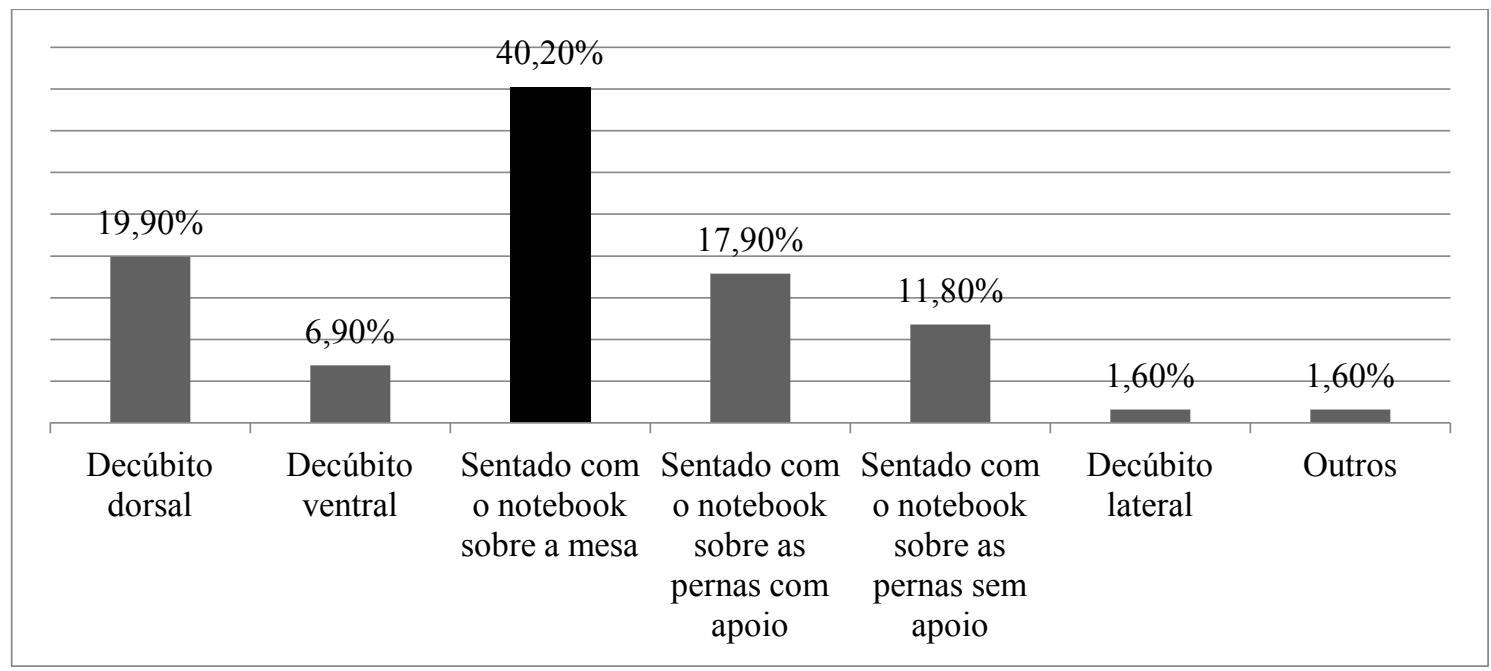

\section{Discussão}

Aalta ocorrência de dor lombar se dá por meio da postura mais utilizada que é apresentada na figura 3. O posicionamento de utilização é relevante para o aparecimento de sintomas de dor, pois a postura mais adotada é o computador sobre a mesa, esse posicionamento quando adotado por longos períodos, é um fator de risco para aumento da sobrecarga na região lombar. Estudos recentes apontam que a postura sentada pode gerar uma sobrecarga maior sobre os discos intervertebrais, gerando desconforto sobre a região da coluna vertebral10,20,24-26.

Em um estudo realizado no Chile ${ }^{1}$, onde foi avaliada a angulação de estruturas corporais durante a atividade de digitação em computadores portáteis, observou-se um grande fator de risco para cervicalgias, ocorrência comprovada com nosso estudo sendo a segunda causa de dor, que podem ser explicadas pela excessiva flexão cervical. Este posicionamento quando adotado por longo tempo, cronicamente pode levar à disfunção da coluna cervical, caracterizando-se por um conjunto de sintomas como dor, limitação da amplitude de movimentos fisiológicos, dor a palpação dos músculos cervicais, desalinhamento das vértebras e até modificação na movimentação normal do sistema craniocervicomandibular gerando ruídos na articulação temporomandibular ${ }^{1,2,15,16,27}$.

As dores em membros superiores nos profissionais da informática foram durante muito tempo as principais queixas, acontecimento relacionado ao uso excessivo dessas extremidades, no entanto é importante fazer uma relação do uso de laptops com aparelhos informatizados de mesa ${ }^{28-32}$. Nas últimas décadas houveram grandes intervenções ergonômicas relacionadas aos usuários que trabalhavam com os computadores convencionais, dessa forma ocorreu a adaptação do ambiente de trabalho, com mesas, cadeiras, posicionamento do monitor e ajuste dos dispositivos periféricos, o que minimizou as queixas relacionadas ao desconforto nas estruturas do corpo, em contramedida a isso, as lesões por esforço repetitivo apresentavam predileção por membros superiores. Tal situação é explicada pela falta de instrução ou corresponsabilização do usuário de fazer pausas e alongamentos, tornando-se necessário a implantação da ginástica laboral com objetivo de minimizar a sobrecarga de estruturas corporais $8,9,21,23$. 
No entanto nossos resultados apontam que os desconfortos em membros superiores, em particular o punho, só apareceram em terceiro lugar no que abrange locais de maior desconforto, confrontando com a literatura que aborda o uso de computadores convencionais. Estudos recentes que avaliam o designer dos computadores laptops, afirmam que apesar da sua portabilidade, seu uso interfere na biomecânica da postura, sendo relevantes dois fatores como: adoção de posturas ergonomicamente erradas geradas pela fácil portabilidade é outro fator relacionado ao desenho do aparelho, que tem a tela junto ao teclado, assim, dificultando a angulação da visão para a tela ou a altura dos membros para digitação ${ }^{1,4,11,14,33}$. Sendo assim, justifica-se o maior porcentual de acometimentos sobre a coluna vertebral em contramedida aos punhos como apresentados na figura 1.

No que se abrange a postura de utilização do computador notebook, pode-se observar o posicionamento como um agravante para o surgimento de dores. Estudos ergonômicos afirmam que quanto menos ergonômica a postura adotada, maior será a ocorrência de dores ${ }^{25,31,34,35}$. Nos resultados encontrados, foi visto que os pesquisados, em maior proporção tendem a adotar um posicionamento biomecânico mais correto, com notebooks sobre uma mesa e o usuário na postura sentada. Entretanto como esses dispositivos apresentam a tela junto ao teclado, limitam a altura da tela, fazendo com que advenha uma maior flexão da cervical desencadeando sobrecarga na musculatura cervical e dor ${ }^{1,4}$. Além disso, a postura sentada quando adotada por longos períodos, pode sobrecarregar a coluna vertebral, causando dor inicialmente, que pode progredir se tiver a somatória de outros fatores de risco como, assento sem apoio e torções de tronco. Fazendo com que se manifeste a hérnia de disco, repercutindo em grande déficit funcional10,13,14,21,30,36.

O tempo que cada pesquisado permanecia usando o computador portátil também foi analisado, uma vez que a literatura aborda que quanto maior o tempo mantido em posturas estáticas, maior é a tendência ao aparecimento de dores, decorrentes da menor oxigenação tecidual| ${ }^{1,3,10,12-14,25,30,33,36}$. O resultado demonstrou que a grande maioria permanecia cerca de uma a duas horas por dia adotando a posição sentada com o computador portátil sobre a mesa, favorecendo a ocorrência de desconforto pela postura adotada, o que se observa na figura 2.

Portanto, com o objetivo de minimizar os impactos relacionados ao uso incorreto dessa tecnologia, tendo em vista as limitações na literatura sobre os aspectos ergonômicos para utilização dos laptops, que ainda hoje não são bem elucidados, sugerimos que os usuários que necessitem utilizar esses aparelhos por tempo prolongado, adotem hábitos posturais biomecânicamente corretos. Como:

Figura 4 - Exemplo de postura biomecânicamente correta durante a utilização prolongada.

\section{Postura incorreta}

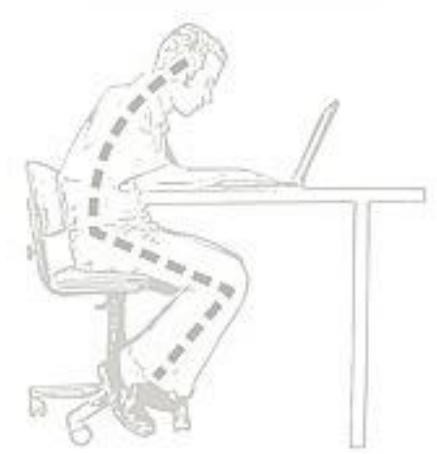

Postura correta

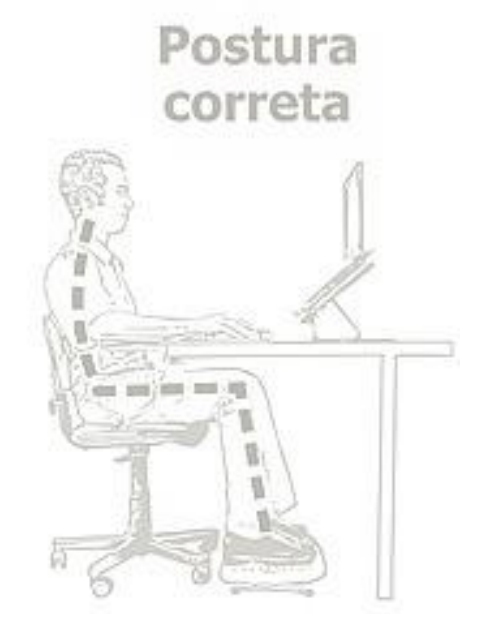

Fonte: Ergonomize.com

- Manter o topo da tela de visualização em aproximadamente ao nível dos olhos (por meio de suporte);

- Fazer pausas frequentes e mudar de postura continuadamente;

- Deixar a cabeça, pescoço e tronco na vertical;

- Permanecer com o tronco perpendicular ao chão;

- Colocar braços e cotovelos junto ao corpo não estendidos para fora;

- Conservar os pulsos e mãos em linha com os antebraços e não descansando na borda do laptop;

- Manter coxas paralelas e pernas perpendiculares ao chão, com os pés totalmente apoiados ao solo;

- Adquirir cadeira ajustável com apoio para as costas e uso de teclado e mouse externo 1,14,17,25,30,31,33-35,37. 


\section{Considerações Finais}

Os resultados sugerem uma provável associação entre dores na coluna e a postura de utilização dos computadores portáteis, que podem se agravar decorrente do tempo de utilização. A maior parte dos entrevistados relatou desconforto musculoesquelético na coluna lombar e coluna cervical, onde o maior tempo de utilização dos computadores portáteis nos acadêmicos está entre 1 a 2 horas. Dentre o posicionamento adotado para utilização dos notebooks, o mais comum é o sentado com o notebook sobre a mesa e em segundo lugar, a posição de decúbito dorsal. Dessa forma, tornase importante criar uma ergonomia para esse tipo de aparelho, principalmente quando o usuário necessitar passar longos períodos de utilização, assim como também é crucial uma corresponsabilidade de adotar hábitos posturais biomecânicamente corretos, assim como pausas e alongamentos, evitando possíveis danos sobre a coluna vertebral.

\section{Referências Bibliográficas}

1. Castellucci I, ZúñigaBenitez L. Postura ,disconfort y productividad durante laejecución de tareas de mecanografíaen computadores personalesportátiles tipo netbook, con y sinmodificaciones ergonómicas. Sigweb, 2009.

2.ChavdaE,ParmarS,ParmarM.Currentpracticeoflaptopcomputerandrelatedhealthproblems:Asurveybasedonergonomics. IntJ MedSciPublicHeal. 2014;3(1):1.

3. Sommerich, C.M.;Starr,H;Smith,C.A.\&ShiversC.Effectsofnotebookcomputerconfigurationandtaskonuserbiomechanics, productivity, andcomfort. Int J IndErgon. 2002;30:7-30.

4. Guimarães GI. Um estudo de caso sobre o uso de computador portátil e implicações ergonômicas em uma universidade brasileira. XVII simpósio Eng produção. 2011;1:1-5.

5. Biswas A LD. ToStudyand Compare theEffectof Laptop Computerswith Desktop ComputersonWorkingPostureand SelfReportedMusculoskeletalSymptoms. Indian J PhysiotherOccupTher. 2012;6(3)

6. IdeWH, Gomes JDO. sinais e sintomas de dort/ler e a eficácia de um programa em fisioterapia preventiva em uma empresa de bebidas - regente feijó / spsignsandsymptomsofdort / ler andeffectivenessofprogram in occupationalphisiotherapy in a beveragecompany - REGEN. 2008;166-80.

7. Jalil S, Nanthavanij S. ANALYTICAL ALGORITHMS FOR ADJUSTING BODY ORIENTATION AND NOTEBOOK COMPUTER SETTINGS TO. 2007;(Ppcoe).

8. Fábri, Tênisson Fernando de S., and Danielle Dias da Silva. "O impacto da ginástica laboral na redução das LER/ DORT." Revista científica do HCE-unidade de emergência. Ano III 2 (2008): 39-48.

9. Falcão, Juliano, et al. "Impactos físicos e mentais de um programa de exercícios terapêuticos direcionado aos servidores de uma instituição pública de Mato Grosso do Sul." Revista Brasileira de Atividade Física \& Saúde 2013,18.2 215.

10. Zemp R, Taylor WR, Lorenzetti S. In vivo spinalpostureduringuprightandreclinedsitting in an office chair. Biomed Res Int $.2013: 916045$.

11. Costa L, XAVIER AADP. Análise da relação entre a postura de trabalho e a incidência de dores nos ombros e no pescoço numa empresa de desenvolvimento e implantação de sistemas de GED. 2006;1-7.

12. Martins, Aline Caldas, and Vanda Elisa AndresFelli. "Sintomas músculo-esqueléticos em graduandos de enfermagem." Enfermagem em Foco 4.1 (2013).

13. Radas A, Mackey M, Leaver A, Bouvier A-L, Chau JY, Shirley D, et al. Evaluationofergonomicandeducationinterventionstoreduceoccupationalsitting in office-baseduniversityworkers: studyprotocol for a randomizedcontrolledtrial. 2013;14(1):330.

14. Asundi K, Odell D, Luce A, Dennerlein JT. Changes in posturethroughthe use ofsimple inclines with notebook computersplacedon a standard desk. ApplErgon 2012 43(2):400-7

15. Nanthavanij S, Jalil S, Ammarapala V. Effectsofbodyheight, notebook computersize, and workstation heightonrecommendedadjustments for properworkposturewhenoperating a notebook computer.J Hum Ergol 2008;37(2):67-81. 16. Leiserson, Dr. "Cervico-cefaleas: diagnóstico y tratamiento." IntraMedJournal 2013; 2.2.

17. Straker L, Skoss R, Burnett A, Burgess-Limerick R. Effectof visual display heightonmodelledupperandlower cervical gravitationalmoment, musclecapacityandrelativestrain. Ergonomics. 2009;52(2):204-21.

18. Pinheiro, F. A., Tróccoli, B. T., \& Carvalho, C. D. Validação do Questionário Nórdico de Sintomas Osteomusculares como medida de morbidade. Rev Saúde Pública, 2002 36(3), 307-12. 
19. Noack-Cooper KL, Sommerich CM, Mirka G a. Collegestudentsandcomputers: assessmentofusagepatternsandmusculoskeletaldiscomfort. Work. 2009 (3):285-98.

20. Peirão R, Tirloni AS, Reis DC dos, Moro ARP. operantes e inoperantes durante. xxviii encontro naceng produção. 2008;1-12. 21. Barros S.S, Ângelo Rdcdo, Uchôa. Lombalgia ocupacional e a postura sentada. Rev dor. 2011;12(3):226-30.

22. Medeiros LGS, almeida costa ML.Alterações musculoesqueléticas e suas implicações na saúde ocupacional. RevBrasEduc e saúde. 2013;(2001):41-7.

23. Santos AC, Bredemeier M, Rosa KF, Amantéa V a, Xavier RM. ImpactontheQualityof Life ofanEducationalProgram for thePreventionofWork-RelatedMusculoskeletalDisorders: a randomizedcontrolledtrial. BMC Public Health .2011;11(1):60.

24. Asundi K, Odell D, Luce A, Dennerlein JT. Changes in posturethroughthe use ofsimple inclines with notebook computersplacedon a standard desk. ApplErgon 2012;43(2):400-7.

25. Alaf A., SamiaA.S ,RahmanA. Cumulativemusculoskeletaldisorderstocomputerproducts use in arabicchildrenandadolescents. Indian J ournalPhysiotherOccupTher. 2010;4(2).

26. Marques NR, Hallal CZ, Gonçalves M. Características biomecânicas, ergonômicas e clínicas da postura sentada : uma revisão. Rev. Fisioterapia e pesquisa 2010;17(3):270-6.

27. Delfino, Paula Domingues, et al. "Cervicalgia: reabilitação; Neckpain: rehabilitation." Acta fisiátrica 2012 19.2.

28. Khan, Rakhshaan, et al. "Knowledgeandpracticesofergonomics in computerusers." JPMA-JournalofthePakistan Medical Association. 2012; 62.3: 213.

29. Zakerian SA, Subramaniam ID. The relationshipbetweenpsychosocialworkfactors, work stress andcomputer-relatedm usculoskeletaldiscomfortsamongcomputerusers in Malaysia. Int J OccupSafErgon. 2009;15(4):425-34.

30. Vilelas, J.M.S; Lopes, S.M.R "o computador eo desenvolvimento de alterações músculo esqueléticas em adolescentes e jovens." Revista Baiana de Enfermagem. 2013;26.2.

31. Shrivastava S, Bobhate P. Computer relatedhealthproblemsamong software professionals in Mumbai: A crosssectionalstudy. Int J HealAlliedSci .2012;1(2):74.

32. Santos P; Rômulo F; Lopes, S.B; Castro R.V. "a relação entre a agenesia do músculo palmar longo e as lers e dorts no punho em alunos universitários da zona oeste do rio de janeiro." Ciência Atual-Revista Científica Multidisciplinar das Faculdades São José. 2013;1.2.

33. Biwas A, Lamba D. Tostudyand compare theeffectof laptop computerwith desktop. Indian J PhysiotherOccup Ther. 2012;6:106. 34. Oliveira LO De, Oliveira JHR De, Godoy LP, Lorenzett DB, Godoy TP. Análise de riscos ocupacionais para implantação de melhorias em uma clínica renal. RevAdm da UFSM .2013;6(4):720-39.

35. Li B, Zhang W, Zhou R, Yang C, Li Z. A comparativeergonomicsstudy: Performingreading-basedtaskson a largescaletabletop vs. laptop. Int J IndErgon. 2012 ;42(1):156-61.

36. Ornelas F.A, Censoni D.C, Zacharias P.D.P, Aprile T.J. Ginastica laboral no setor de informatica: abordagem fisioterapêutica. Revinspirar. 2009;3:35.

37. Sommerich CM, Starr H, Smith CA, Shivers C. Effectsof notebook computerconfigurationandtaskonuserbiomechanics, productivity, andcomfort. Int J IndErgon.; 2002. 30(1):7-31. 


\section{Juscelino Francisco Vilela Junio}

Endereço para correspondência - Rua: Julieta Meira, $n^{\circ}$ 24, Bairro: Centro, CEP: 55370000 , São Bento do Una, PE, Brasil.

E-mail: juscelinovilela@yahoo.com.br

Lattes: http://lattes.cnpq.br/0586321738807927

Anna Luísa Araújo Brito - annaluisa_12@hotmail.com

Juceluce da Silva Vilela - jucelucebio@hotmail.com

Luiz Henrique Ferreira de Aguiar - luiz.aguiarf@gmail.com

Emerson Nunes da Silva - Emersonnunes8@hotmail.com

Eurico Solian Torres Liberalino - euricotorres@asces.edu.br

Enviado em 25 de março de 2015.

Aceito em 22 de outubro de 2015. 
